\title{
CONSTRUÇÃO ESTEREOGRÁFICA SIMPLES PARA LOCALIZAÇÃO DO EIXO ÓTICO DE INDICATRIZ UNIAXIAL*
}

\author{
IBRAHIM OCTAVIO ABRAHĀO ** \\ ARARY MARCONI**
}

\begin{abstract}
RESUMO
listuda-se uma nova e simples contrução estereográfica para localização do eixo ótico de minerais uniaxiais, na platina universal. São usados dois ângulos: $\theta$, escolhido previamente e com rotação no cixo $\mathrm{A}_{4}(\mathrm{E}-\mathrm{W})$ da platina universal c $\Psi$, ângulo de exłinção medido na platina do microscópio. O método sugere a claboração de uma tabcla, baseada em valores $\theta$ с $\Psi$.
\end{abstract}

\section{INTRODUÇÃO}

$\mathrm{Na}$ prática petrográfica, é muito freqüente a necessidade de determinar a posição do eixo ótico de um mineral uniaxial em secção delgada. Essa necessidade pode estender-se a um grande número de grânulos, como é o caso de estudos petrográficos, em que a orientação dos grânulos em relação a uma determinada direção é estabelecida.

O presente trabalho trata de uma construçâo estereográfica muito simplęs que permite a localização do eixo ótico mediante a medição de um único ângulo.de extinção.

\section{REVISÃO DA LITERATURA}

A deierminação da posição do eixo ótico de um mineral uniaxial não oferece, na realidade, maiores dificuldades na piatina universal. O procedimento clássico é o descrito por MUIR (1967): o mineral é levado a uma posição de extinçâo e se assegura estar seu eixo ótico no plano vertical de traço $\mathrm{N}-\mathrm{S}$. Após uma rotação de $45^{\circ}$, efetua-se, na posição diagonal, numa rotação $\mathrm{em} \mathrm{A}_{4}$ no sentido em que a birrefringência diminui. Quando esta se anula, o mineral estará con o eixo coincidindo com a vertical. Essa consirução, embora simples e segura, esbarra em um inconveniente sério: a rotação até a birrefringência nula só é exequível dentro das limitações da platina. Quando o eixo é inacessivel, torna-se necessário optar por outro sistema, como o de levar o eixo ótico à horizontal e controlar sua posição pela máxima cor de interferência.

Os autores não conhecem referência a construções como a que aqui propõem. Com efeito, numerosos trabalhos especializados e minuciosos, como JOHANSENN (1918). ROSENBUSCH e WÜLFING (1924), BEREK (1924), NIKITIN (1936), KERR (1959), WAHLSTROM (1969), BLOSS (1970), entre outros, referem-se, no máximo, ao que descreve Muir. Por outro lado, há um considerável número de trabalhos sobre a localização de eixos óticos de minerais biaxiais, através de construções mais ou menos compli-

* Entregue para publicação em 13/10/1975.

** Departamento de Solos e Geologia, LSALQ - USP. 
cadas (TOCHER, 1962, 1964; JOEL e MUIK, 1957; JOEL, 1964; WRIGHT, 1966; ABRAHÃO, 1968, 1974). Essas construçōes referem-se a operações executadas na platina universal ou no "spindle stage".

CHOMARD (1934) e ABRAHÃO e MARCONI (1975) estudam a aplicação das rotações de Euler na localização do eixo ótico de minerais uniaxiais. Chomard mostra que o problema é resolvido por uma equação do 20 grau, de cujas raízes uma apenas corresponderia à solução do problema. Abrahão e Marconi demonstram que ambas as raízes correspondem a posições possiveis do eixo ótico e indicam processos que permitem optar por uma das raízes.

\section{FUNDAMENTO TEÓRICO}

Dada uma secção de um mineral uniaxial na platina universal, cujo eixo ótico está em qualquer posição (exceto em 0 ou $\alpha$ ) há uma rotação ao redor do eixo do microscópio que a leva à extinção, sobre xx' ou yy'. Consideremos toda secção em extinção com o pólo de seu eixo ótico sobre xx', posição que sempre é facilmente alcançada.

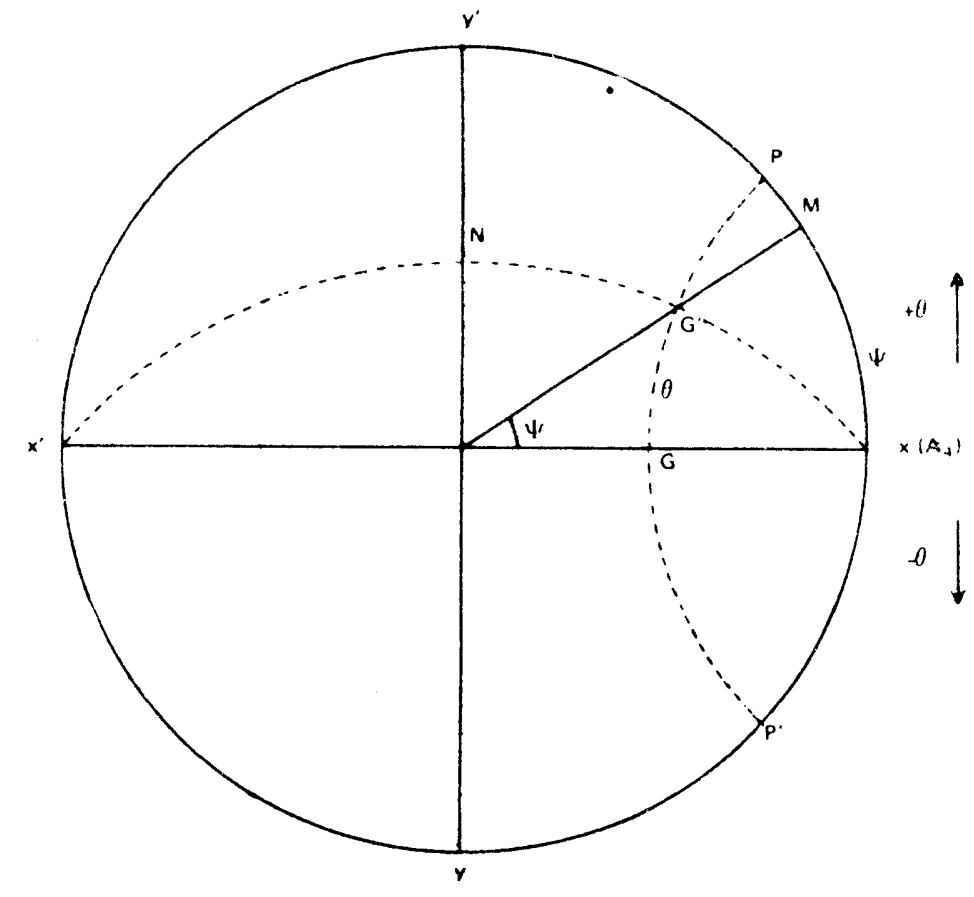

Fig. 1

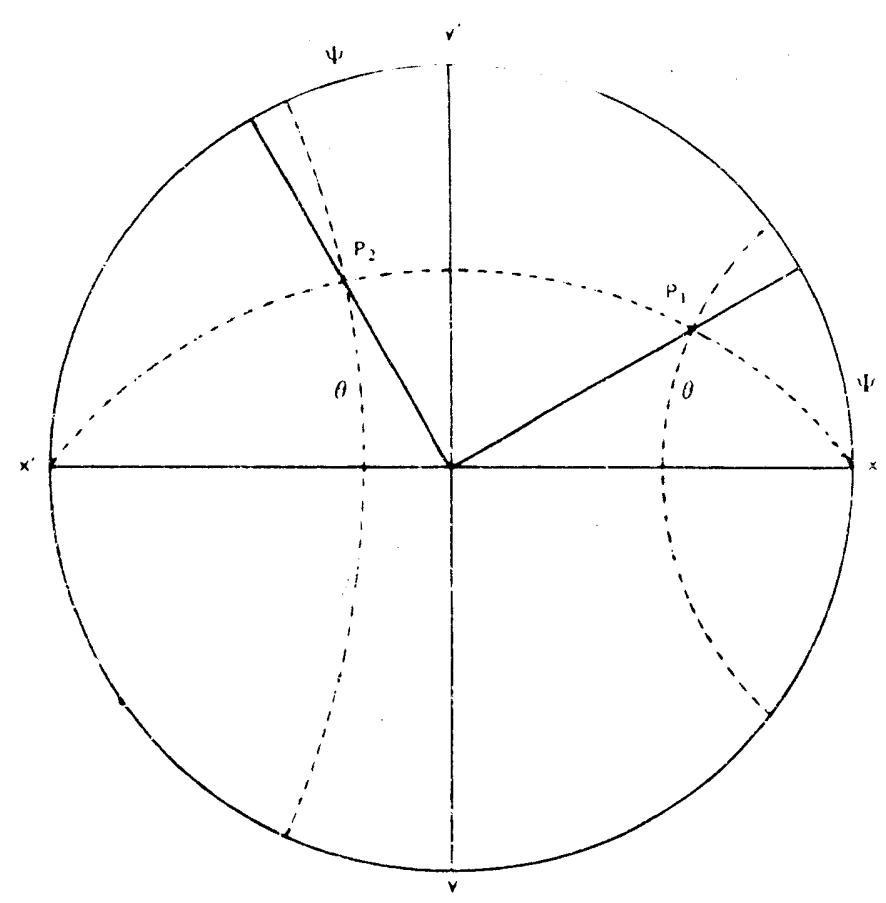

Fig. 2

Façamos sobre $\mathrm{G}$ (fig. 1) uma rotação 0 ao redor de $\mathrm{A}_{4}$, positiva para a frente. $\mathrm{O}$ pólo do eixo ótico percorre o paralelo PGP', assume a posição G' e sai de extinção, que pode ser restituida por uma rotação $\Psi$ na platina do microscópio. Observa-se, pois, que a cada $\theta$ corresponde um $\Psi$. Se são conhecidos, portanto, os àngulos $\Psi$ (medido) e $\theta$ (escolhido) o ângulo $\delta$ entre o eixo ótico e Oz pode ser determinado.

Realmente, G' é a interseç̧ão da direção $\mathrm{OM}$ com o meridiano de traço $\mathrm{xN}^{\mathrm{N}}$ ', de inclinação $\theta$, que contém, necessariamente $G^{\prime}$. 
Há, entretanto, duas posição de $G$ sobre $x x^{\prime}$ que conduzem à mesma rotação $\Psi$ após a rotação $\theta$. É o caso das secções de pólos $P_{1}$ e $P_{2}$ da fig. 2 , conforme já se demonstrou em trabalho anterior (ABRAHÃO e MARCONI, 1975). A decisão entre $P_{1}$ e $P_{2}$ é muito simples: o aumento de $\Psi$ para qualquer rotação $\theta$ positiva, indica que trata-se de $P_{1}$ e a diminuição que é $\mathrm{P}_{2}$.

Essa construção sugere a elaboração de uma tabela $\theta, \Psi, \delta$. Com efeito para um $\theta$ fixo (fig. 3), tomemos todos os infinitos pontos sobre $x x^{\prime}$ e marquemos sobre o meridiano $\mathrm{xNx}$ ', a posição de todos os pontos rebatidos. Sendo, $\mathrm{xNx}$, o lugar geométrico desses pontos, basta ler para cada um deles o respectivo ângulo de extinção em projeção este-

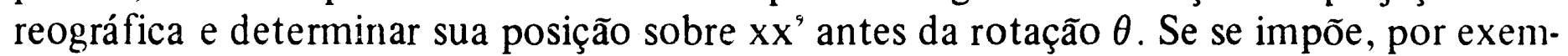
plo, a divisão de $x \mathrm{Nx}^{\prime}$ de grau em grau, a tabela teria 180 posições fixas de $\delta$ (inteiras) e os respectivos valores de $\Psi$ (lidos). À guisa de exemplo, apresenta-se uma seqüência de alguns valores da tabela, com os respectivos pares de mesma extinção para $\theta=30^{\circ}$, ..๊nsiderada a precisão de leitura de $0,25^{\circ}$ na rede de Wulff.

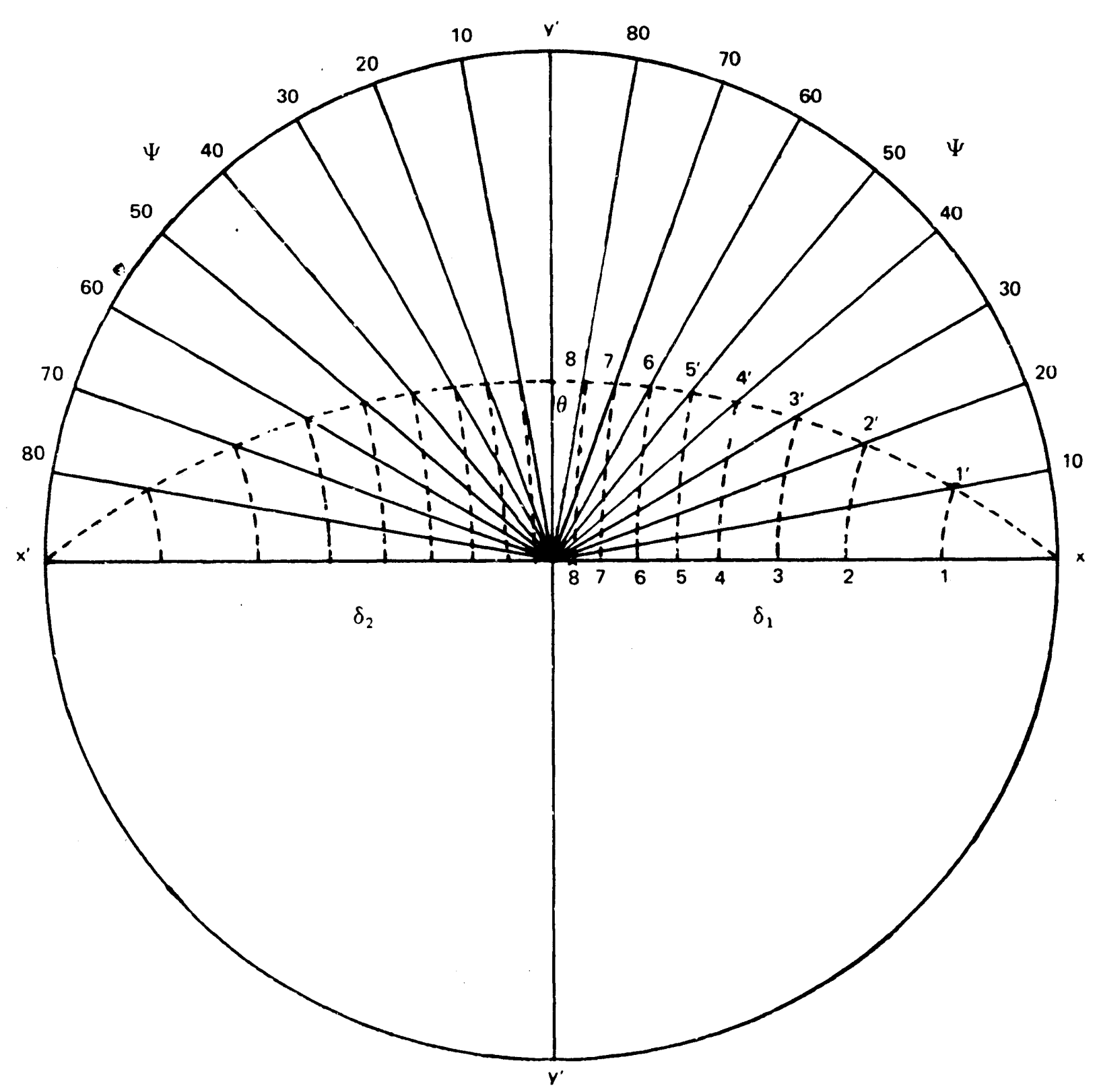

Fig. 3 


\begin{tabular}{crrr}
\hline Pólo & $\Psi$ & $\delta_{1}$ & $\delta_{2}$ \\
\hline 0 & 0.00 & 90.00 & 0.00 \\
1 & 5.00 & 80.00 & 2.50 \\
2 & 10.25 & 70.00 & 5.25 \\
3 & 16.00 & 60.00 & 8.00 \\
4 & 22.25 & 50.00 & 11.50 \\
5 & 30.75 & 40.00 & 16.75 \\
6 & 40.75 & 30.00 & 23.25 \\
7 & 54.00 & 20.00 & 34.50 \\
8 & 70.25 & 10.00 & 54.25 \\
9 & 0.00 & 0.00 & 90.00 \\
\hline
\end{tabular}

\section{CONCLUSÕES}

É possivel, com uma construção estereogrática muito simples, determinar a posição do eixo ótico de um mineral uniaxial. Isa-se, apenas, un angulo 0 escolhido previamente e a única medição necessária é a de um ângulo de extinção.

Sugere-se que, usando-se essa construção, seja elaborada uma tabela, que simpiificaria ainda mais o trabalho de rotina.

\section{SUMMARY}

\section{SIMPLE: STEREOGIRAPIIC CONSTRUCTION FOR LOCATING; THI: OPTICAL AXIS ()F UNIAXIAL INI)ICATRIX}

A new and simple stereographic construction for locating the optical axis of uniaxial minerals in the universal stage is presented. Two angles are used: 0 , previously selected and rotated in the $\mathrm{A}_{4}(\mathrm{E}-\mathrm{W})$ axis of the universal stage; and $\psi$. extinction angle measured in the stage of the microscope. The procedure suggests the elaboration of a table. based on 0 . $\Psi$ values.

\section{LITERATURA CITADA}

ABRAHÃ(). 1.O.. 1968. Contribuição ao lintudo do Método Analítico de Chomard. Tese Livre-Docencia. ISALQ. I SP. Piracicaba. SP, $132 \mathrm{pp}$.

ABRAHĀO. I.O.. 1974. Determinacão de $2 \mathrm{~V}$ de Playioclásios pelo Método Analítico com Valores de OVlultiplos de $45^{\circ}$. Anais da I:SALQ. LSP. 31: 19-33. 
ABRAHÃO, I.O. c MARCONI, A., 1975. Aplicação do Método Analítico à Indicatriz Uniaxial. Anais da ESALQ, USP, 32.

BEREK, M., 1924. Mikroskopiche Mineralbestimmung mit Hilfe der Universaldrehtischmethoden. Berlin, Verlag von Gebrüder Borntraeger, 168 p.

BLOSS, F.D., 1970. Introduction a los Metodos de Cristalografia Óptica. Ed. Omega S.A., Barcelona, Espanha, 320 p.

CHOMARD, L., 1934. Théoric et Pratique de la Méthode Fédorow. Procedé Classique et Méthode Analy tique Générale. Annales des Mines, Paris, V: 153-218.

JOEL, N., 1964. A Stereographic Construction for Determining the Optic Axis of a Biaxial Crystal Directly from a Few Lxtinction Measurements. Min. Mag., 33: 769-779.

JOEL, N. e MUIR, I.D., 1957. New Techniques for the Universal Stage. I. An Extinction Curve Method of Determination of the Optical Indicatrix. Min. Mag., 31:860-877.

JOHANSENN, A., 1918. Manual of Petrographic Methods. McGraw Hill Book Co., Inc.. USA, 2 a ed., 649 p.

KERR, P.I., 1959. Optical Mincralogy. McGraw Hill Book Co., Inc. USA, 3ạ ed., 442 p.

MUIR, I.D., 1967. Microscopy: Transmitted Light. Physical Methods in Determinative Mineralogy, ed. J. Zussman, Academic Press, London, 31-102.

NIKITIN, W., 1936. Die Fedorow-Methode. Berlin, Verlag von Gebrüder Borntraeger, 109 p.

ROSINBUSCH, H. e WÜliFING, E.A.. 1921. Mikroskopische Physiographic der Petrographisch Wichtigen Mineralien. Stuttgart, E. Schweizerbart'sche Verlagsbuch handlung. 846 p.

TOCHI:R, F.I:., 1962. Extinction Curves and 2V: a Stereographic Solution. Min. Mag.. 33:52-58.

TOCHI:R, F.E., 1964. Ixtinction and 2V:a Simple Stcregraphic Solution of General Application. Am. Min., 49: 1622-1630.

WAHLSTROM, ミ.I:., 1969. Cristalografia Óptica. Livro Técnico S.A., Rio de Janciro, RJ, 367 pp.

WRIGHT, H.G., 1966. Determination of Indicatrix Orientation and $2 \mathrm{~V}$ with the Spindle Stage: a Caution and a Test. Am. Min., 51:919-924. 
